\title{
Effets de la convection thermocapillaire sur les instabilités de la phase fluide et l'interaction solide/liquide en croissance dirigée
}

\author{
El Alami Semma ${ }^{1,2, a}$, Mohammed El Ganaoui ${ }^{3}$, Rachid Bennacer $^{4}$, \\ Abdelkhalek Cheddadi ${ }^{1}$ et Patrick Bontoux ${ }^{2}$ \\ 1 École Mohammadia d'Ingénieurs, Université Mohammed V, Avenue Ibn Sina, Agdal, Rabat, Maroc \\ 2 MSNM UMR CNRS 6181 La Jetée, Technopôle de Château-Gombert, 38 rue Frédéric Joliot-Curie, \\ 13451 Marseille Cedex 20, France \\ 3 SPCTS, UMR CNRS 6638, Université de Limoges, 123 Albert Thomas, 87000 Limoges, France \\ 4 LEEVAM-LEEE, 5 mail Gay Lussac, Université de Cergy Pontoise, 95031, France
}

Reçu le 8 octobre 2003, accepté le 30 janvier 2004

\begin{abstract}
Résumé - Ce travail concerne l'étude des instabilités convectives et l'interaction solide/liquide pour une configuration de croissance dirigée horizontale d'un matériau à faible nombre de Prandtl $(\operatorname{Pr}=0,015)$. Les configurations de ce type ont fait l'objet de plusieurs études restreintes à la phase fluide. Il s'agit ici d'étudier les transitions au sein du bain fondu en présence de la convection thermocapillaire et l'interaction avec le front. L'interaction se focalisant dans cette étude sur les aspects convectifs sans prise en compte des effets de la chaleur latente. L'approche de résolution est basée sur la méthode de localisation du front couplée à une approximation de type volumes finis. La convection thermocapillaire agissant dans le même sens que la convection thermogravitationnelle amorti les oscillations introduites par la convection thermogravitationnelle. D'autre part, l'effet de la vitesse de translation de l'ampoule pour le contrôle du processus est également analysé.
\end{abstract}

Mots clés : Croissance cristalline / convection thermocapillaire / méthode enthalpique / convection oscillatoire / volumes finis

Abstract - Effects of the thermocapillary convection on the flow stability and the interaction with solid/liquid interface in directional solidification. This work concerns the study of the convective instabilities interacting with the solid/liquid interface for a horizontal Bridgman configuration filled with a low Prandtl number material $(\operatorname{Pr}=0.015)$. This kind of configuration was the subject of several studies restricted to fluid phase. We study here the transitions to oscillatory regimes in the presence of thermocapillary convection and the interaction with the solidification front. This interaction focuses here on the convective aspects without considering the latent heat effects. A computational model based on the enthalpy method and a finite volumes approximation is adapted to the phase change problem. The computed results indicate that the surface tension can play a stabilising role on the flow regime. In the same way, the growth rate can also contribute to control the directional process.

Key words: Crystal growth / thermocapillary convection / enthalpy method / oscillatory convection / finite volumes

\section{Introduction}

Le développement important des applications a créé une forte demande en matériaux semi-conducteurs et une exigence sévère de leur qualité. Ces cristaux sont

\footnotetext{
a Auteur correspondant : semmaalam@yahoo.fr
}

produits à partir de bains fondus en utilisant des techniques de croissance cristalline diverses (configuration de Bridgman, Zone Flottante, Czochralski, ...). Lors de la croissance cristalline par la technique de Bridgman horizontale $(\mathrm{BH})$, des gradients de température suffisamment élevés peuvent se produire par conduction dans le bain fondu. Ces gradients thermiques sont à l'origine de 
forces de gravité qui engendrent des mouvements convectifs importants au sein du fluide. Ces mouvements de convection constituent un mode de transfert d'énergie important qui peut accélérer le processus et affecter significativement la qualité du cristal (Brice [1], Monberg [2], Potard et al. [3], Timchenko et al. [4]).

La convection dans le fluide peut être générée par des forces de volume induites par les gradients thermiques ou les gradients solutaux dans le fluide (convection naturelle), ou par des forces de surface causées par la variation de la tension superficielle qui résulte de la variation de la température ou de la concentration à la surface libre. L'étude des effets simultanés de la convection gravitationnelle et de la convection thermocapillaire en présence de changement de phase a fait l'objet de plusieurs études. Il a été montré que l'association des deux modes d'écoulement peut affecter la forme ainsi que la progression de l'interface solide/liquide (Keller et Bergman [5], Bergman et Webb [6], Lacroix [7], Sampath et Zabaras [8]). Cet effet dépend, évidemment, du rapport des forces de volume et de surface (Zebib et al. [9]).

Par ailleurs, les travaux étudiant la transition des écoulements aux régimes instables en configurations à surface libre sont souvent restreints à des configurations purement fluides (Pulicani et al. [10], Ben Hadid et Roux [11]). Cependant, la présence d'un front de solidification dont la position et la forme évoluent de manière transitoire affectent nettement les transitions dans les configurations BH (Zhou et Zebib [12], Lan et al. [13], El Ganaoui et al. [14], Semma et al. [15]).

L'objectif du présent papier est d'analyser l'effet de la convection thermocapillaire et de la vitesse de tirage sur le régime oscillatoire de l'écoulement et son interaction avec l'interface solide/liquide. La phase fluide est caractérisée par un faible nombre de Prandtl $(\operatorname{Pr}=0,015)$. La chaleur latente du matériau est négligée et la surface libre est supposée non déformable. Lorsque la convection thermocapillaire domine la convection gravitationnelle, l'interface subit des déformations importantes et le champ thermique dans la région de changement de phase est stratifié. Pour les régimes oscillatoires où la convection gravitationnelle est dominante, la convection thermocapillaire agissant dans le même sens que la convection de volume constitue un mécanisme stabilisateur de l'écoulement. Ces observations sont valables pour les deux types de conditions aux limites thermiques sur les parois horizontales (adiabatiques ou conductrices). L'effet de la vitesse de tirage sur les régimes instables a été aussi analysé pour le cas adiabatique. Le tirage dans les deux sens, permet d'amortir les oscillations de l'écoulement par rapport à la configuration statique.

\section{Formulation mathématique}

Le système physique considéré (Fig. 1) est une cavité rectangulaire de longueur $L$ et de hauteur $H(A=L / H=$ 5 ) et remplie d'un matériau de faible nombre de Prandtl $(\operatorname{Pr}=0,015)$. Les parois verticales sont isothermes et soumises respectivement aux températures froide $T_{\mathrm{F}}$ et

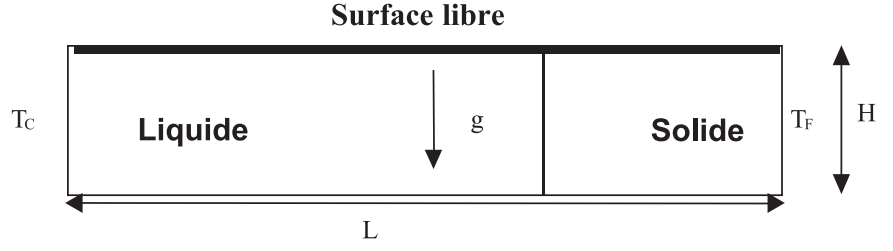

Fig. 1. Configuration géométrique.

chaude $T_{\mathrm{C}}$ encadrant la température de fusion $T_{\mathrm{f}}$. Des cas de parois horizontales adiabatiques (A) ou conductrices (C) sont examinés.

La surface supérieure est supposée libre, plane et indéformable et elle est soumise à la tension superficielle $\sigma$ supposée varier linéairement avec la température suivant la relation $\sigma=\sigma_{0}\left(1-\gamma\left(T-T_{0}\right)\right), \sigma_{0}$ étant la tension superficielle à la température de référence $T_{0}$ et $\gamma=-\frac{1}{\sigma_{0}}\left(\frac{\partial \sigma}{\partial T}\right)_{0}$ le coefficient thermique de tension superficielle. Ce coefficient est positif pour les semi-conducteurs car ceux-ci se caractérisent par une tension superficielle qui décroît avec la température.

Le modèle mathématique repose sur l'hypothèse d'un fluide newtonien en écoulement laminaire. Le matériau est supposé pur, homogène et isotrope à propriétés physiques constantes sauf la densité vérifiant l'hypothèse de Boussinesq $\rho=\rho_{0}\left(1-\beta\left(T-T_{0}\right)\right)$.

Le modèle mathématique repose également sur une formulation de type enthalpie adaptée aux problèmes de croissance dirigée (El Ganaoui et al. [14]). Les équations de conservation de la quantité de mouvement et de l'énergie sont reformulées de façon à prendre en compte les phénomènes relatifs au changement de phase solideliquide par l'introduction des termes d'homogénéisation et sont ainsi résolues sur l'ensemble du domaine physique.

La chaleur latente durant le changement de phase est introduite dans l'équation de l'énergie en utilisant l'enthalpie sensible définie par :

$$
h=C p T+f_{\mathrm{l}} L_{\mathrm{f}}
$$

avec $L_{\mathrm{f}}$ la chaleur latente de fusion, $C p$ la chaleur spécifique (supposée identique dans les deux phases) et $f_{1}$ la fraction massique de la phase liquide déterminée à partir de la température du matériau par la relation ciaprès :

$$
\begin{cases}f_{\mathrm{l}}=0, & T \leq T_{\mathrm{f}}-\varepsilon \\ f_{\mathrm{l}}=\frac{T-T_{\mathrm{f}}}{2 \varepsilon}+\frac{1}{2} & T_{\mathrm{f}}-\varepsilon<T<T_{\mathrm{f}}+\varepsilon \\ f_{\mathrm{l}}=1 & T \geq T_{\mathrm{f}}+\varepsilon\end{cases}
$$

où $2 \varepsilon$ est un intervalle représentant la taille thermique de la zone de régularisation due au changement de phase solide/liquide prise égale à $1 \%$ de la température de fusion et validée sans effets significatifs sur les résultats.

Le problème est réduit en utilisant comme valeurs de référence de la longueur, du temps, de la vitesse et de la température respectivement $H, H^{2} / \nu, \nu G r^{0,5} / H$ et 
$\delta T=\left(T_{\mathrm{c}}-T_{\mathrm{f}}\right) /(A-1)$. Les paramètres adimensionnels résultants sont : les nombres de Grashof $(G r=$ $\left.g \beta \delta T H^{3} / \nu^{2}\right)$, de Prandtl $(\operatorname{Pr}=\nu / \alpha)$, de Stefan $($ Ste $=$ $\left.C p \Delta T / L_{\mathrm{f}}\right)$ et de Peclet $\left(P e=u_{\mathrm{t}} \cdot G r^{-0,5} H / v\right)$ avec $u_{\mathrm{t}}$ la vitesse de tirage de l'ampoule suivant la direction horizontale.

Le système d'équations adimensionnelles est écrit suivant la formulation adoptée par El Ganaoui [16] :

$$
\begin{aligned}
& \nabla \cdot \mathbf{u}=0 \\
& \frac{\partial \mathbf{u}}{\partial t}+G r^{0,5}(\mathbf{u} \cdot \nabla) \mathbf{u}=-\nabla p+\nabla^{2} \mathbf{u}+G r^{0,5} T \mathbf{e}_{\mathrm{z}} \\
& -G r^{-0,5} \frac{H^{2}}{K\left(f_{\mathrm{l}}\right)}\left(\mathbf{u}-\mathbf{u}_{\mathrm{t}}\right) \\
& \frac{\partial h}{\partial t}+S t e^{-1} \frac{\partial f_{1}}{\partial t}=\frac{1}{\operatorname{Pr}} \nabla^{2} T-G r^{0,5}\left(u-u_{\mathrm{t}}\right) \cdot \nabla h
\end{aligned}
$$

La perméabilité $K$ est évaluée par la formule de Carman-Kozeny dont la validité a été démontrée pour l'écoulement laminaire d'un fluide visqueux dans un milieu poreux (voir par exemple Beckermann et al. [17]) :

$$
K=K_{0}\left[\frac{f_{1}^{3}}{\left(1-f_{1}\right)^{2}}\right]
$$

Les conditions aux limites dynamiques :

$\mathbf{u}(0, y)=\mathbf{u}(A, y)=\mathbf{u}(x, 0)=\mathbf{u}_{\mathrm{t}}$ sur les parois rigides.

$\frac{\partial u}{\partial y}(x, 1)=f_{1} \frac{R e}{G r^{0,5}} \frac{\partial \theta}{\partial x}(x, 1), \quad v(x, 1)=0$ sur la surface libre.

avec Re le nombre de Reynolds-Marangoni défini comme étant le rapport du nombre de Marangoni $M a=$ $\frac{|\partial \sigma / \partial T| \Delta T H}{\mu}$ au nombre de Prandtl : $R e=M a / \operatorname{Pr}$ et $\mu$ la viscosité dynamique de la phase fluide. $u$ et $v$ sont, respectivement, les composantes du vecteur vitesse $\mathbf{u}$ suivant les directions horizontale $x$ et verticale $y$.

Les conditions aux limites thermiques:

$T(0, y)=A-1, T(A, y)=-1$ sur les parois verticales.

$T(x, 0)=T(x, 1)=(A-1)-x$ parois horizontales conductrices : (C).

$\frac{\partial T}{\partial y}(x, 0)=\frac{\partial T}{\partial y}(x, 1)=0$ parois horizontales adiabatiques : (A).

\section{Approximations numériques}

Le système d'équations de conservation (3)-(5) est approché numériquement à l'aide d'une méthode de volumes finis. Les termes diffusifs sont discrétisés par le schéma de différences centrées. Les termes convectifs sont discrétisés à l'aide d'un schéma implicite de type ULTIMATE2D qui associe un schéma convectif décentré quadratique avec une stratégie de limitation de flux
(Hayase et al. [18], Leonard [19]). Le couplage vitessepression est résolu en utilisant l'algorithme SIMPLEC (Van Doormal et Raithby [21]). Un schéma implicite de Crank-Nicolson est utilisé pour la discrétisation des dérivées temporelles. La présente méthode est d'ordre 2 en espace et en temps. Ce modèle numérique a montré une bonne aptitude dans la résolution des problèmes instationnaires avec changement de phase dans différentes configurations (Semma et al. [15]). Un maillage de $140 \times 70$ dans le domaine $(x, y)$ a été retenu à la suite d'une étude de maillage assurant l'indépendance des résultats de simulation vis-à-vis des paramètres de discrétisation. Ce maillage est également raffiné du côté de la surface libre pour bien tenir compte de la profondeur de la surface de Marangoni et dans la zone de mobilité de l'interface solide/liquide pour encadrer la région de changement de phase. Le pas de temps est considéré dans l'intervalle $\left[2 \times 10^{-5}, 10^{-4}\right]$ suivant la raideur numérique du cas traité.

\section{Résultats et discussion}

Dans la présente étude, la chaleur latente est considérée nulle $\left(S t e^{-1}=0\right)$ afin de focaliser notre analyse sur les effets de la convection thermocapillaire et de la vitesse de translation de l'ampoule sur le diagramme de bifurcation.

\subsection{Régime stationnaire}

Dans un premier temps l'interactions de la convection thermocapillaire avec le champ thermique et en particulier l'interface solide/liquide en régime stationnaire a été considérée pour un faible nombre de Grashof $G r=1000$. Le choix de cette valeur est adopté pour se placer dans des conditions de microgravité et assurer une dominance des forces de surface sur celles d'origine volumique pour des Re élevés. La configuration est supposée statique (pas de translation $P e=0$ ).

En absence de la tension superficielle, l'ensemble du domaine est occupé par une cellule de convection (Fig. 2a). Pour $0<R e<10^{3}$, cette cellule est scindée en une cellule principale du côté de l'interface solide/liquide et une cellule secondaire contra-rotative du côté de la paroi chaude. Au centre du bain fondu, il y a apparition d'un vortex de faible dimension attaché à la paroi horizontale du bas. En augmentant $R e$, la cellule de convection du coté de l'interface devient de plus en plus concentrée entraînant une déformation importante de l'interface solide/liquide (Fig. 3). La cellule secondaire contra-rotative augmente aussi avec $R e$ en s'étendant vers la paroi chaude. Pour $R e=7 \times 10^{4}$, il y a apparition d'une deuxième cellule secondaire contra-rotative, la première cellule secondaire augmente d'intensité et se déplace du côté de la paroi chaude (Fig. 2b). En augmentant davantage la valeur de $R e$ à $3 \times 10^{5}$, l'écoulement dans la phase fluide devient contrôlé principalement par la variation de la tension superficielle à la surface libre. Ceci explique le changement significatif au niveau de 


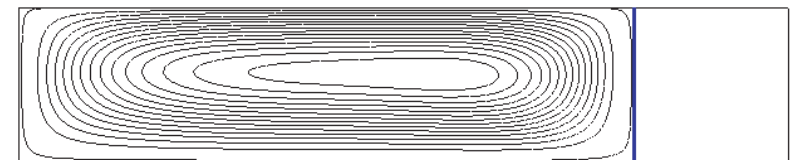

(a)

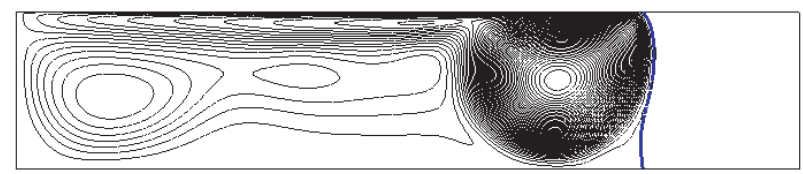

(b)

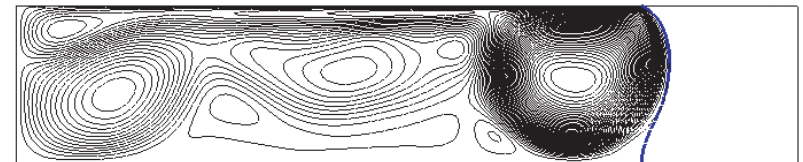

(c)

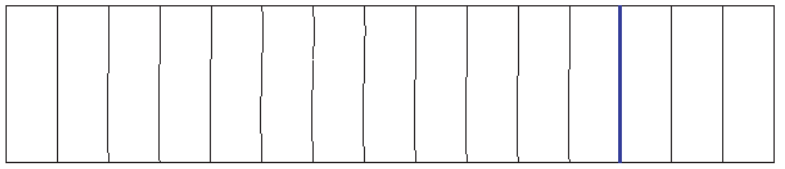

(d)

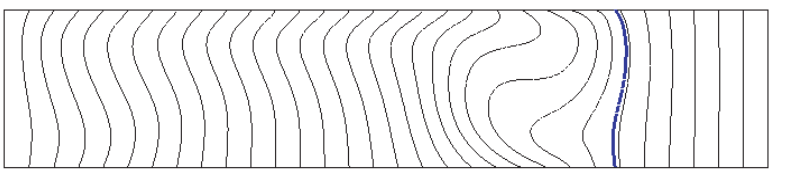

(e)

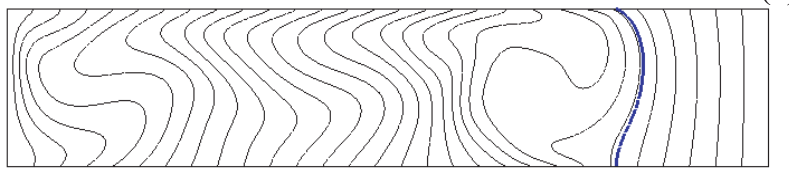

(f)

Fig. 2. Structures d'écoulement et isothermes pour différentes valeurs de $\operatorname{Re}(G r=1000)$ : pour $\operatorname{Re}=0, \psi_{\max }=0,171, \psi_{\min }=0$, $\Delta \psi=0,0107 ; R e=7 \times 10^{4}, \psi_{\max }=13,51, \psi_{\min }=-2,019, \Delta \psi=0,243 ; R e=3 \times 10^{5}, \psi_{\max }=29,783, \psi_{\min }=-10,262$, $\Delta \psi=0625$.

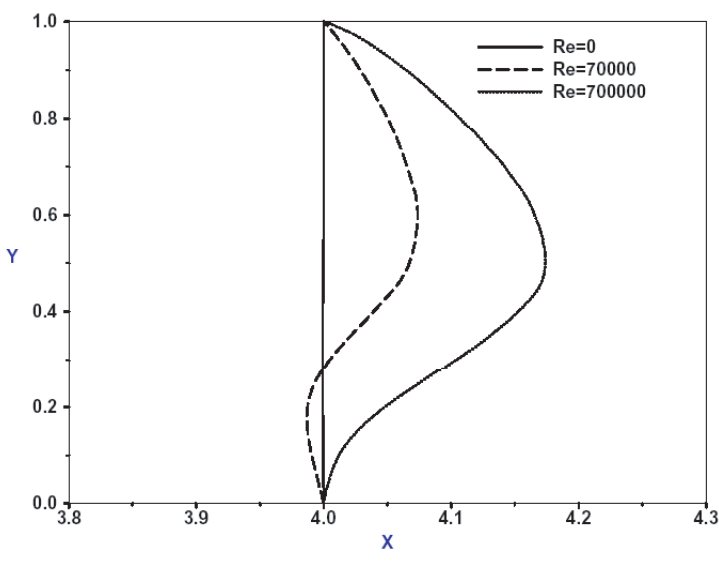

Fig. 3. Forme de l'interface solide/liquide en régime permanent pour trois nombres de Reynolds $(G r=1000)$.

la structure d'écoulement qui devient multicellulaire (Fig. 2c). Le vortex contra-rotatif du côté de la paroi chaude augmente davantage et entraîne une stratification des lignes isothermes dans cette zone (Fig. 2f). La vitesse axiale à la surface libre atteint sa valeur maximale au voisinage du point singulier du côté du front de solidification et augmente considérablement avec $R e$, la dynamique liée à ce problème spécifique est détaillée dans [22].

\subsection{Régime oscillatoire}

\subsubsection{Effet de la convection thermocapillaire}

Dans le cas à surfaces horizontales conductrices $(\mathrm{C})$, et pour un nombre de Reynolds-Marangoni nul $(R e=$ $0)$, l'écoulement présente pour les faibles nombres de Grashof une seule cellule de convection. En augmentant le nombre de Grashof, la cellule se scinde en deux cellules co-rotatives composées d'un vortex principal du côté de

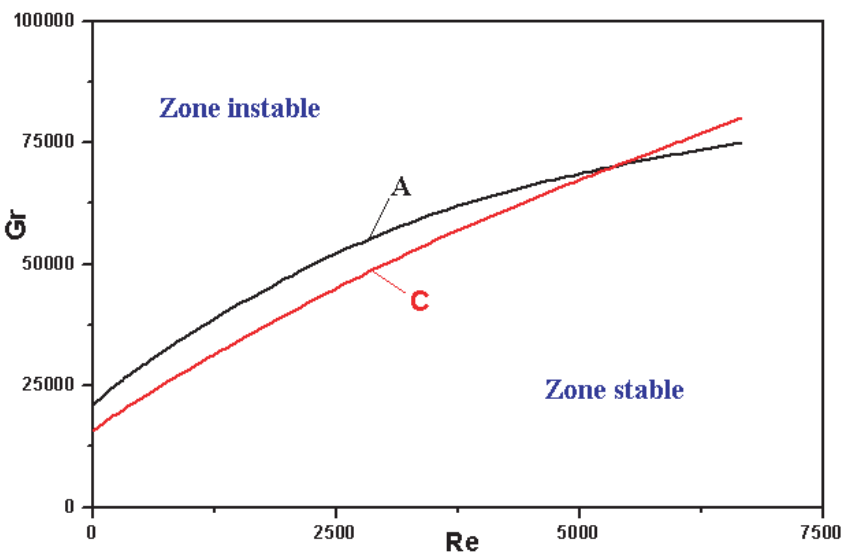

Fig. 4. Diagramme de stabilité du système dans les deux cas : Adiabatique (A) et Conducteur (C).

l'interface de solidification et d'un vortex secondaire du côté de la zone chaude. Lorsque $G r$ atteint un seuil critique autour de $G r_{\mathrm{C}}=15750$, l'écoulement devient oscillatoire monopériodique avec une fréquence de l'ordre de $f=13,6$. Cette fréquence augmente avec $G r$. Lorsqu'on augmente $R e$, la région stable s'élargit et la transition vers le régime oscillatoire est réalisée pour des nombres de Grashof plus importants (Fig. 4).

Pour illustrer l'effet de la tension superficielle sur le régime oscillatoire, $G r$ est fixé à 40000 et $R e$ varie $\left(0<R e<2 \times 10^{3}\right)$. La figure 5 présente, pour trois nombres de Reynolds, l'évolution temporelle de la déformation de l'interface $\lambda$ ( $\lambda$ représentant la différence entre les deux limites extrêmes atteintes par l'interface suivant la direction $x$ ). L'augmentation de l'intensité des forces thermocapillaires réduit l'amplitude d'oscillation de la déformation de l'interface et de l'intensité de l'écoulement dans le bain fondu du système. Pour $R e=2000$, le régime d'écoulement devient stationnaire 


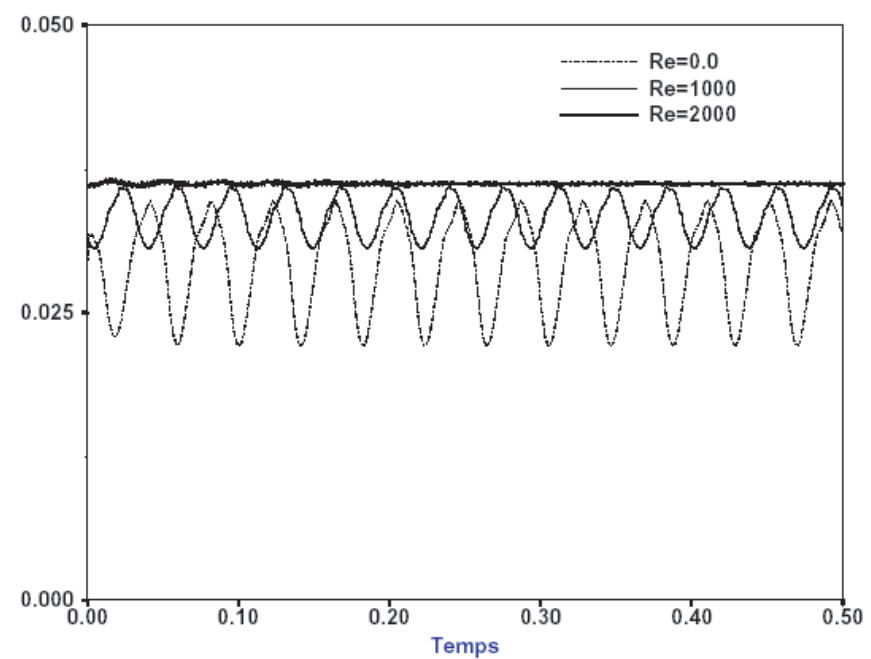

Fig. 5. Évolution temporelle de la déformation de l'interface $\lambda$ pour différentes valeurs de $\operatorname{Re}(G r=40000)$.

(Fig. 5). Cependant, la fréquence des oscillations ainsi que la déformation moyenne augmentent avec Re. Dans le cas adiabatique (A), l'évolution de la structure de l'écoulement pour un nombre de Reynolds-Maragoni nul $(R e=0)$ est qualitativement la même que celle décrite cidessus pour le cas $(\mathrm{C})$. Cependant l'écoulement bénéficie d'une bande de stabilité plus large en raison de la relaxation introduite par le gradient thermique à la surface qui n'est plus constant mais s'adapte au régime d'écoulement au sein du bain fondu. Ainsi, la transition aux régimes instationnaires est obtenue pour un nombre de Grashof de l'ordre de $G r_{\mathrm{C}}=21000$. Ces transitions sont en bon accord en ordre de grandeur avec l'étude d'une phase fluide similaire [23].

Lorsqu'on applique des forces thermocapillaires $(R e \neq$ 0 ), le régime oscillatoire de l'écoulement est amorti et le passage aux régimes instationnaires est réalisé pour des nombres de Grashof plus importants (Fig. 4). Cette transition se fait pour des nombres de Grashof moins importants pour $R e>5000$ en comparaison avec le cas de conditions conductrices. Ceci peut être attribué à la relaxation du bain fondu introduite par le déplacement de l'interface solide/liquide dans le cas adiabatique. La figure 6 présente la structure d'écoulement et les isothermes pour le régime stationnaire correspondant à $R e=10^{4}$ et $G r=75000$. La structure d'écoulement est composée de trois cellules co-rotatives. La troisième cellule apparaissant au sein de la zone chaude est due à l'élargissement de la taille du bain fondu sous l'effet de l'association des forces thermocapillaires et thermogravitationnelles. Les isothermes sont stratifiées du coté de la surface libre. Cette stratification est plus nette dans la région de changement de phase ainsi que dans les espaces occupés par les cellules de convection. Néanmoins, on note que la présence de la tension superficielle à la surface libre entraîne une augmentation nette du volume du bain fondu et l'interface solide/liquide subit des déformations importantes.

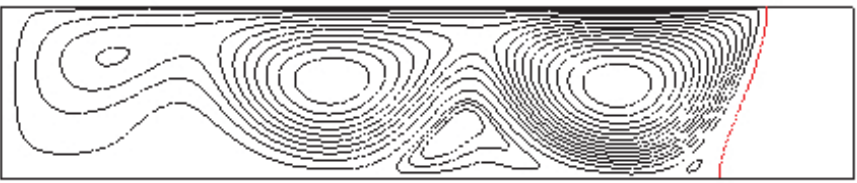

(a)

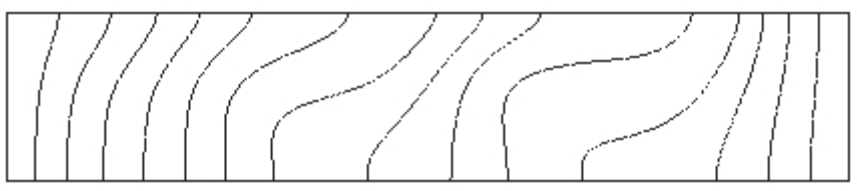

(b)

Fig. 6. Structure d'écoulement (a) et lignes isothermes (b), $G r=75000$ et $R e=10^{4}$.

\subsubsection{Effet de la vitesse de tirage}

Le tirage est un procédé externe qui permet de contrôler le déplacement de l'ampoule dans la zone de gradient thermique. Ce facteur régit la cinétique de changement de phase et influence le régime oscillatoire de l'écoulement. Dans le cas d'une cavité de Bridgman verticale (Benielli et al. [20]) ont montré expérimentalement et numériquement que la vitesse de tirage affecte la nature de l'écoulement au voisinage de l'interface par l'apparition de cellules secondaires pour des vitesses de tirage importantes. Ces cellules augmentent la concavité du front de solidification.

Dans la suite l'influence de ce paramètre sur le comportement oscillatoire de l'écoulement est étudiée, nous avons considéré le cas simple où la convection thermocapillaire est négligeable $(R e=0)$. Le nombre de Grashof considéré est $G r=24000$, valeur proche de la limite seuil de transition instationnaire $\left(G r_{\mathrm{C}}=21000\right)$.

La figure 7 présente l'évolution temporelle du maximum de la fonction de courant pour $G r=24000$. Le tirage affecte les vitesses au sein du bain fondu et par conséquent, le mode oscillatoire de l'écoulement. Les oscillations de l'écoulement sont amorties par rapport au cas statique. Le seuil de transition aux régimes instationnaires est décalé et la zone stable est élargie. Afin de quantifier l'effet de la vitesse de tirage sur le régime d'écoulement et l'interaction avec l'interface solide/liquide, on a considéré $G r=30000$ valeur pour laquelle le régime oscillatoire devient plus sévère, et on a présenté sur la figure 8 la variation de la valeur moyenne du maximum de la fonction de courant et de la déformation $\lambda$ en fonction $\mathrm{du} P e$. On remarque que la valeur moyenne de la fonction de courant varie linéairement avec le nombre de Peclet avec un point d'inflexion localisé en un Peclet proche de 0. Elle subit une augmentation de $31 \%$ lorsque la Peclet passe de 0,1 à $-0,1$. Les pentes des comportements de $\psi_{\max }$ moy en fonction de $P e$ sont $-0,84$ en solidification et $-1,04$ en fusion. Cette variation s'effectue dans le sens inverse de la variation de la déformation moyenne de l'interface qui augmente de $13 \%$ lorsque le Peclet passe de $-0,1$ à 0,1 . Les deux courbes présentent un 


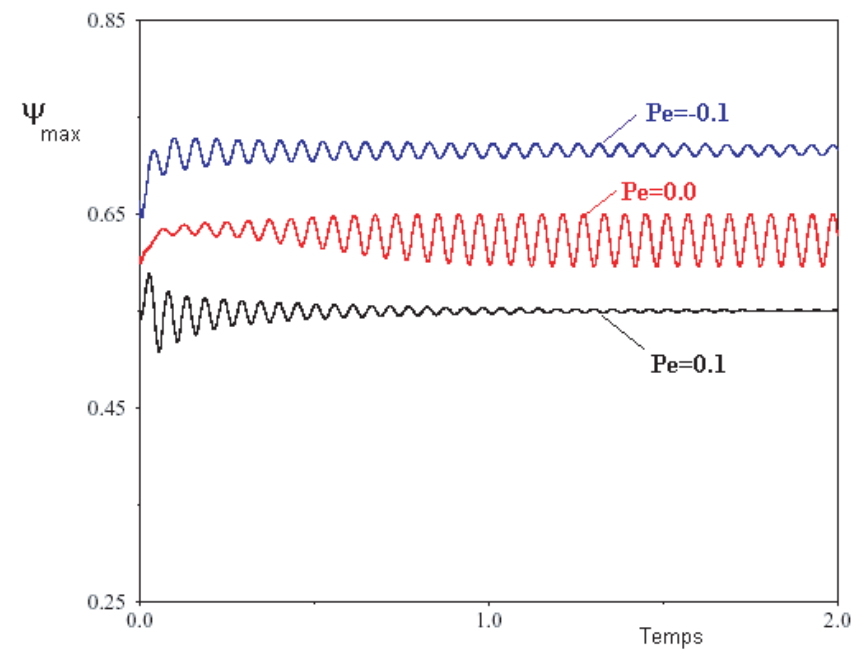

Fig. 7. Effet de la vitesse de tirage sur l'évolution temporelle de $\psi_{\max }(G r=24000)$.

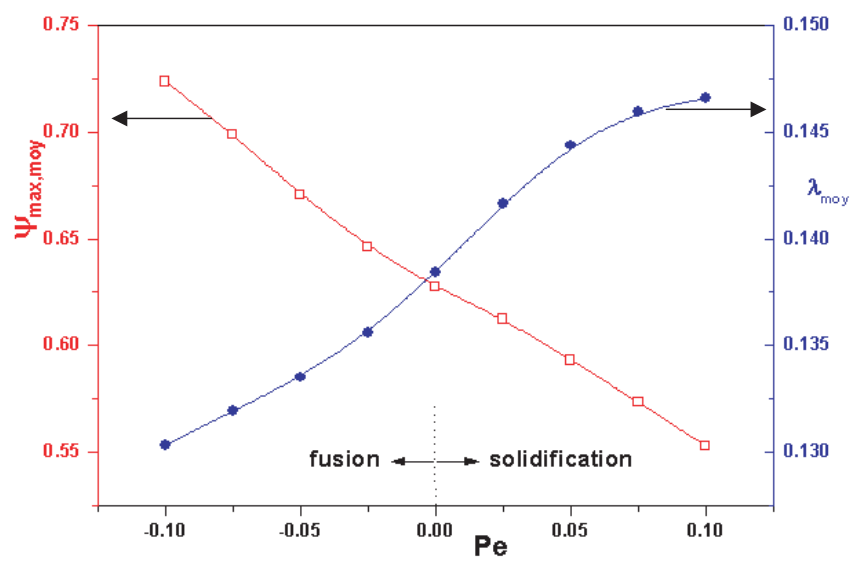

Fig. 8. Effet de la vitesse de tirage sur les valeurs moyennes de $\psi_{\max }$ et la déformation $\lambda(G r=30000)$.

même point d'inflexion pour lequelle les amplitudes d'oscillation de la fonction de courant et de la déformation de l'interface atteignent leurs valeurs maximales (Fig. 9). La vitesse de tirage amorti les fluctuations de l'interface qui sont réduites de $50 \%$ lorsque la vitesse de tirage passe de 0 à 0,1 (Fig. 9).

Ce travail constitue une première approche de l'effet de quelques paramètres de contrôle (tirage et convection thermocapillaire) sur les instabilités convectives en croissance dirigée horizontale. Cette étude sera étendue pour illustrer l'influence de ces paramètres sur la macroségrégation dans le cas des matériaux binaires en régimes instables.

\section{Conclusion}

Dans le présent travail, la convection thermocapillaire couplée à la convection naturelle et agissant dans le sens coopératif $(R e>0)$ est étudiée pour une configuration de Bridgman horizontale à faible nombre de Prandtl

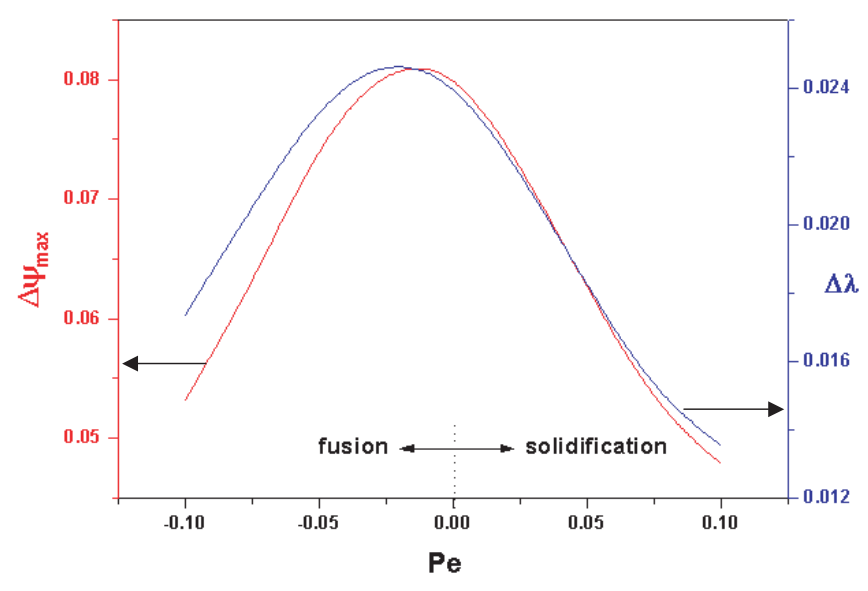

Fig. 9. Effet de la vitesse de tirage sur l'amplitude d'oscillation de $\psi_{\max }$ et de la déformation $\lambda(G r=30000)$.

$(\operatorname{Pr}=0,015)$. Les résultats numériques montrent que la forme de l'interface solide/liquide est largement affectée lorsqu'on se place dans des conditions de microgravité où les forces de surface sont dominantes. Cependant, la présence de la convection thermocapillaire peut améliorer la stabilité de l'écoulement et retarder le seuil de la transition vers le régime oscillatoire déclenché par la dominance des forces gravitationnelles. Nous avons aussi montré que le tirage de l'ampoule dans le four amorti les oscillations de l'écoulement dans le bain fondu. Ce paramètre constitue donc un mécanisme important pour contrôler la stabilité de l'écoulement en croissance dirigée horizontale. Néanmoins il faut signaler que les vitesses de tirage importantes avantageront l'apparition d'instabilités d'ordre morphologique. Concernant la surface libre, les investigations présentées ici comme extension des résultats de référence sans changement de phase négligent les échanges convectifs avec le gaz, ces derniers sont à prendre en compte lors de simulation réalistes de la configuration de Bridgman horizontale.

\section{Références}

[1] J.C. Brice, The growth of crystals from liquids. NorthHollands, New York, 1976

[2] E. Monberg, in: D.T.J. Hurle (ed.), Handbook of Crystal Growth 2A: Basic Techniques, North-Holland, Amsterdam 52, 1994

[3] C. Potard, P. Dussert, T. Duffar, Cryst. Res. Technol. 32 (1997) 925

[4] V. Timchenko, P.Y.P. Chen, E. Leonardi, G. de Vahl Davis, R.A. Abbaschian, Computational study of transient plane front solidification of alloys in a Bridgman apparatus under microgravity conditions, Int. J. Heat and Mass Transfer 43(6) (2000) 963-980

[5] J.R. Keller, T.L. Bergman, Prediction of conjugate heat transfer in a solid liquid system: Inclusion of buoyancy and surface tension forces in liquid phase, J. Heat Transfer 111 (1989) 690-698

[6] T.L. Bergman, B.W. Webb, Simulation of pure metal melting with buoyancy and surface tension forces in the 
liquid phase, Int. J. Heat Mass Transfer 33(1) (1990) 139-149

[7] M. Lacroix, Effects of buoyancy and surface tension forces on the melting of a metal, Num. Heat Transfer 19A (1991) 101-115

[8] R. Sampath, N. Zarabas, Numerical study of convection in directional solidification of binary alloy driven by the combined action of buoyancy, surface tension, and electromagnetic field, J. Comp. Physics 168 (2001) 384-411

[9] A. Zebib, G.N. Homsy, E. Meiburg, High Marangoni number convection in a square cavity, Phys. Fluids 28 (1985) 3467-3476

[10] J.P. Pulicani, E.C. Del Arco, A. Randriamampianina, P. Bontoux, R. Peyret, Int. J. Methods fluids 10 (1990) 481

[11] H. Ben Hadid, B. Roux, Buoyancy and thermocapillary driven flows in a shallow open cavity: Unsteady flow regimes, J. Crystal Growth 97 (1989) 217-225

[12] H. Zhou, A. Zebib, Oscillatory convection in solidifying pure metals, Num. Heat Transfer 22A (1992) 435-468

[13] C.W. Lan, M.K. Chen, M.C. Liang, Bifurcation and stability analyses of horizontal Bridgman crystal growth of a lox prandtl number material, J. Crystal Growth 187 (1998) 303-313

[14] M. El Ganaoui, P. Bontoux, D. Morvan, Localisation d'un front de solidification en interaction avec un bain fondu instationnaire, C. R. Acad. Sci. Paris, 306, II b (1999), 41-48

[15] E. Semma, M. El Ganaoui, A. Cheddadi, P. Bontoux, Étude numérique des instabilités de la phase fluide et de l'interface de solidification en croissance dirigée horizontale, C. R. Acad. Sci. Paris, 331 (2003) 631-639
[16] M. El Ganaoui, Modélisation numérique de la convection thermique instationnaire en présence d'un front de solidification déformable. Application à la croissance cristalline, thèse de doctorat, Université d'Aix Marseille II, 1997

[17] Beckermann, R. Viskanta, S. Ramadhyani, Natural convection in vertical enclosures containing simultaneously fluid and porous layers, J. Fluid Mech. 186 (1988) $257-284$

[18] T. Hayase, J.A.C. Humphrey, R. Greif, A consistently formulated Quick scheme for fast and stable convergence using finite-volume iterative calculation procedures, J. Comp. Phys. 98 (1992) 108-118

[19] B.P. Leonard, The ULTIMATE conservative difference scheme applied to unsteady one-dimensional advection, Computer Methods in Applied Mechanics and engineering 88 (1991) 17-74

[20] D. Benielli, M. El Ganaoui, E. Semma, N. Bergeon, H. Jamgotchian, P. Voge, B. Billia, P. Bontoux, Effect of thermal convection on directional solidification of succinonitrile- acetone alloys: comparison between experimental and numerical studies, J. Phys. IV France 11 (2001) Pr6135-Pr6142

[21] P. Van Doormaal, G.D. Raithby, Enhancement of SIMPLE Method for predicting incompressible fluid flow, Num. Heat Transfer 7 (1984) 147-163

[22] Canright, Phys. Fluids 6(4) (1994) 1415-1424

[23] M. Mundrane, J. Xu, A. Zebib, Thermocapillary convection in a rectangular cavity with a deformable interface, Advances in Space Research 16(7) (1995) $41-53$ 